\title{
Diagnóstico y medicación: la práctica médica en el Hospital Psiquiátrico del Valle entre 1956-1970 ${ }^{1}$
}

\author{
José Fernando Sánchez Salcedo ${ }^{2}$ \\ Universidad del Valle, Cali, Colombia \\ jose.sanchez@correounivalle.edu.co \\ Recibido: 15 de enero de 2016 \\ Aceptado: 1. de junio de 2016 \\ Disponible en línea: 20 de diciembre de 2016
}

1 Este artículo es producto del avance del proyecto de investigación: "Del Asilo al Hospital. Procesos de institucionalización de la salud mental en Bogotá y Cali, 1940-1970” (2015), realizado con la profesora María del Carmen Castrillón y financiado por la Vicerrectoría de Investigaciones de la Universidad del Valle (C.I. 6175).

2 Doctor en Ciencias Políticas y Sociales: Sociología, Universidad Católica de Lovaina (Bélgica). Profesor Asociado, Departamento de Ciencias Sociales, Facultad de Ciencias Sociales y Económicas, Universidad del Valle (Cali, Colombia). 


\title{
Diagnóstico y medicación: la práctica médica en el Hospital Psiquiátrico del Valle entre 1956-1970
}

\section{Resumen}

A mediados del siglo XX, se lleva a cabo en Colombia un cambio institucional en el diagnóstico y el tratamiento de la locura. Surgen nuevas concepciones sobre la enfermedad mental, y se crean departamentos de Psiquiatría, tanto en las antiguas como en las recién inauguradas facultades de Medicina, que implementan nuevos tratamientos, influenciados por la Psiquiatría norteamericana. Siguiendo una línea de investigación historiográfica, el presente trabajo busca describir, a través de la revisión de 308 historias clínicas como fuente principal, el tipo de diagnósticos y el tratamiento utilizado en el proceso de transición del Asilo de Cali a hospital psiquiátrico, ocurrido entre 1956 y 1970. Entre los principales hallazgos se destaca la institucionalización de un conjunto de prácticas médicas que revela una clara influencia de la Psiquiatría norteamericana. En lo que respecta al tratamiento, las historias muestran el uso recurrente de nuevos fármacos antipsicóticos, combinados con terapias de electrochoque, ergoterapia y psicoterapia.

Palabras clave: cambio institucional; Psiquiatria; diagnósticos; terapias psiquiátricas

\section{Diagnosis and Medication: The Medical practice in the Psychiatric Hospital of the Valle 1956-1970}

\begin{abstract}
At the middle of the twentieth century, an institutional change in the diagnosis and treatment of insanity took place in Colombia. New conceptions of mental illness arose and psychiatry departments were created, both in old and in newly opened medicine faculties, which implemented new treatments influenced by American psychiatry. Following a line of historiographic research, this work seeks to describe, through the review of 308 medical histories as the main source, the type of diagnoses and the treatment used during the process of transition of Cali's Asylum to become a psychiatric hospital, between 1956 and 1970 . Prominent among our main findings is the institutionalization of a set of medical practices that reveals a clear influence of North American Psychiatry. With regard to treatment, the stories show the frequent use of new antipsychotic drugs, combined with electroshock therapy, occupational therapy and psychotherapy.
\end{abstract}

Keywords: institutional change; psychiatry; diagnostics; psychiatric therapies

\section{Diagnóstico e medicação: a prática médica no Hospital Psiquiátrico de o Valle entre 1956-1970}

\begin{abstract}
Resumo
Em meados do século XX tem lugar na Colômbia uma mudança institucional no diagnóstico e tratamento da loucura. Há novas concepções sobre a doença mental e são criados departamentos de Psiquiatria, tanto nas antigas quanto as recém-inauguradas escolas de medicina que implementam novos tratamentos, influenciados pela Psiquiatria norteamericana. Seguindo uma linha de pesquisa historiográfica, este trabalho tem como objetivo descrever, através da revisão de 308 prontuários como fonte principal, o tipo de diagnóstico e de tratamento utilizado no processo de transição do Asilo de Cali para Hospital psiquiátrico, ocorrido entre 1956 e 1970. Entre os principais achados aparece a institucionalização de um conjunto de práticas médicas que revela uma clara influência da Psiquiatria norte-americana. No que diz respeito ao tratamento, as histórias mostram o uso recorrente de novos medicamentos antipsicóticos, combinado com terapia de eletrochoque, ergoterapia e psicoterapia.
\end{abstract}

Palavras-chave: mudança institucional; Psiquiatria; diagnósticos; terapias psiquiátricas 


\section{Introducción}

Entre 1950 y 1960 se construyó un hospital psiquiátrico moderno en la ciudad de Cali, que sustituyó al Asilo San Isidro. Este último había sido creado en 1937 como correccional y, posteriormente, en 1940, fue habilitado como manicomio. Este cambio institucional se dio en un periodo de importantes transformaciones que modificaron las concepciones sobre la locura, su diagnóstico y tratamiento. Entre los aspectos más importantes que caracterizan este periodo están: 1) la nueva concepción sobre la enfermedad mental que acuñó la Organización Mundial de la Salud (OMS) en 1950, enfatizando en el carácter preventivo y la necesidad de integrar la práctica psiquiátrica a las demás ramas de la Medicina; y 2) la influencia de la Psiquiatría norteamericana en la reestructuración de las instituciones mentales, a través de: la formación de especialistas en el país; el establecimiento de nuevas formas de clasificación de las enfermedades mentales, derivadas de un enfoque psicodinámico que combinó el uso de fármacos y la terapia psicoanalítica; el desarrollo de un proceso de desinstitucionalización del enfermo mental, a fin de reducir los costos de los tratamientos, y finalmente, la ampliación de nuevos servicios, como el Hospital día y la consulta externa para la atención a la población.

Todos estos aspectos influenciarian, en 1955, la práctica médica del Departamento de Psiquiatría adscrito a la Facultad de Medicina de la Universidad del Valle. Esta última fue creada en 1950, y al igual que otras instituciones y facultades de Medicina del país contó con el respaldo de la Fundación Rockefeller, que garantizó no solo apoyo económico, sino un importante direccionamiento académico para la formación de médicos psiquiatras en universidades norteamericanas.

El presente estudio busca dar cuenta de estos cambios a partir del análisis de historias clínicas, como el principal instrumento de registro y de diagnóstico que implementaron los médicos psiquiatras del Asilo, en el proceso mismo de su transición hacia hospital moderno. Dicho trabajo supuso la revisión y sistematización de 308 historias clínicas del periodo comprendido entre 1956 y $1970^{3}$, que fueron recogidas del

\footnotetext{
Es importante mencionar el carácter no probabilístico de esta muestra, pues su objetivo es ofrecer una mirada más cualitativa de las historias clínicas producidas durante el periodo estudiado.
} 
Archivo del Hospital Psiquiátrico San Isidro. Con la información obtenida se originó una base de datos en la que se privilegiaron aspectos relacionados con el diagnóstico y el tratamiento recibido por los pacientes, a través de la consulta externa y la hospitalización. Se tuvieron en cuenta, además, otros datos como: sexo, edad, parentesco de quienes llevaban al paciente a consulta y tipo de atención prestada.

El artículo se divide en cuatro partes. En la primera, se proponen los referentes conceptuales desde los cuales se abordará el estudio. En la segunda, se lleva a cabo una contextualización histórica del proceso de transformación del Asilo a hospital psiquiátrico, entendido como un cambio institucional en el que se resalta la conformación de un campo organizacional que posibilita dicho cambio. En la tercera parte, se hace una presentación de las historias clínicas como fuente para la investigación historiográfica y se exponen los principales hallazgos obtenidos de su análisis. Finalmente, en la cuarta, se proponen algunas conclusiones.

\section{La dinámica institucional}

Los estudios sobre la historia de la salud mental y de la Psiquiatría han estado fuertemente influenciados por el pensamiento de autores como Foucault (1961), Goffman (1972), Basaglia (1978), entre otros. No obstante, como lo afirma Huertas (2012), "es posible establecer una historia de la psiquiatría antes y después de Foucault" (p. 13). Su análisis de las instituciones y principalmente del Estado como uno de los principales impulsores del confinamiento masivo de locos, ha influenciado la mayoría de los estudios sobre el Estado en América Latina a finales del siglo XIX y principios del XX. En dichos estudios se concibe este entramado institucional "como un Estado normalizador, con varios frentes de acción: penitenciario, judicial, educativo, sanitario, etc." (Bohoslavky, 2005, p. 50).

Esta caracterización reforzó una cierta creencia sobre la inmensa capacidad de intervención de los aparatos institucionales para modificar - domesticar- la conducta de los sujetos subalternos, 
al punto de generar un sobredimensionamiento del control y del poder del Estado, que hizo perder de vista sus particularidades históricas y, por ende, su desarrollo desigual en los diferentes países latinoamericanos. Un problema adicional de esta lectura es que redujo las instituciones a la imagen de simples máquinas para conformar, disciplinar y destruir toda individualidad.

A partir de 1990 hay una renovación del estudio de las instituciones, las politicas públicas y del Estado, que genera nuevas lecturas y perspectivas teóricas y metodológicas sobre las organizaciones sociales en disciplinas como la Economía, la Ciencia Política y la Sociología. En uno de los textos emblemáticos del neoinstitucionalismo, Powell y DiMaggio (1999) plantean que el proceso de "burocratización y otras formas de homogenización surgen de la estructuración de los campos organizacionales (Giddens, 1979), y son efectuados fundamentalmente por el Estado y las profesiones, [...] grandes racionalizadores de la segunda mitad del siglo XX" (p. 105). En este sentido, Rivas (2003) señala que "Las instituciones articulan y desarticulan, estructuran y desestructuran, integran y desintegran, promueven y restringen nuestras conductas, nuestras reglas, códigos, tradiciones, etc.” (p. 40).

Aunque el neoinstitucionalismo abarca diversas perspectivas -económica, política, histórica y sociológica-, sus autores coinciden en definir las instituciones "como las reglas de juego en una sociedad o, más formalmente, los constreñimientos u obligaciones creados por los seres humanos que le dan forma a la interacción humana" (North, 1990, p. 3).

Estos patrones sociales tienden a reproducirse, porque tienen que ver con la manera en que los individuos hacen las cosas. "Por tanto en la vida cotidiana los hombres actúan como si siguieran reglas; saben cómo hacer las cosas y ese saber incluye una experiencia práctica, una experiencia reflexiva y una orientación normativa" (Escalante, 1992, p. 30). El origen de estos patrones de comportamiento es de gran importancia histórica, porque contribuye a estructurar la política, tanto a nivel de las estrategias como de los objetivos mismos y 
las posibilidades de consenso y conflicto. A este respecto, Powell y DiMaggio (1999, p. 18) expresaron:

El nuevo institucionalismo aborda lo histórico como un proceso de cambio institucional continuo, en el que desde la división más elemental del trabajo, hasta la constitución de los Estados modernos, se han establecido cuerpos cada vez más complejos de rutinas de comportamiento o reglas de juego que surgen para reducir la incertidumbre existente en la interacción entre los entes sociales [...] la repetición durante periodos prolongados en el tiempo de estas rutinas constituye el mundo de las instituciones.

Para comprender el cambio institucional, es preciso identificar el tipo de actores que existen en la sociedad y la relación que establecen con su entorno institucional. En este estudio se abordará, por un lado, el campo organizacional que define la posibilidad de un cambio institucional, y por otro, el papel que juegan en dicho proceso de cambio las transformaciones en los sistemas de creencias.

El campo organizacional lo conforman "aquellas organizaciones que, en conjunto, constituyen un área reconocida de la vida institucional: los proveedores principales, los consumidores de recursos y productos, las agencias reguladoras y otras organizaciones que dan servicios o productos similares" (Powell y DiMaggio, 1999, p. 106). Un campo se estructura en cuatro etapas, tal y como lo señalan Powell y DiMaggio (1999, p. 106):

[...] aumento en el grado de interacción entre las organizaciones en el campo; surgimiento de estructuras interorganizacionales de dominio y de patrones de coalición claramente definidos; incremento de la carga de información de la que deben ocuparse las organizaciones que participan en el campo; y desarrollo de la conciencia entre los participantes de un conjunto de organizaciones que están en una empresa común.

La importancia del concepto de campo para el análisis del paso del Asilo a hospital, radica en que busca privilegiar los vínculos entre las 
organizaciones como un factor central para explicar el proceso de institucionalización del Hospital como una organización moderna. Así mismo, el enfoque en los sistemas de creencias logra mostrar la función que cumplen las nuevas concepciones de la enfermedad y del diagnóstico médico en la consolidación de una práctica médica, entendida como un conjunto de actividades y procedimientos que se van haciendo rutinarios, hasta convertirse en reglas.

Los campos organizacionales se encuentran enmarcados en sistemas de creencias, cuyas ideas influyen "en la forma como los actores perciben sus intereses y opciones en primera instancia, incluyendo las instituciones que preferirian continuar y mantener bajo circunstancias diversas" (Campbell citado por Muñoz, 2014, p. 409). La adscripción en un sistema de creencias permite aglutinar a las organizaciones a partir de puntos de vista e intereses comunes, generando de esta forma procesos de homogenización. Este proceso - en el que las organizaciones buscan parecerse a otras cuando comparten las mismas condiciones ambientales-, ha sido definido por Powell y DiMaggio (1999) como isomorfismo.

\section{La constitución del Hospital Psiquiátrico San Isidro}

\section{Caracteristicas del contexto}

La transformación del Asilo de locos en hospital psiquiátrico fue llevada a cabo en un periodo de importantes cambios políticos, económicos y sociales como: la retoma del gobierno por parte de los conservadores en las elecciones de 1946; el recrudecimiento de la violencia politica que entre 1946 y 1953 dejó 140.000 víctimas; el golpe de Estado que realizaron los militares en 1953; y el advenimiento, a finales de esta década, de una nueva coalición política, el Frente Nacional, que ofreció una paz relativa a la violencia partidista a través de un modelo político excluyente. Todos estos acontecimientos se desplegaron en un contexto de crecimiento económico y de desarrollo de la industrialización en el país. 
A nivel local, Cali había empezado, desde mediados de la década del treinta, un proceso de industrialización que se incrementó entre 1944 y 1955. "En 1934 se fundaron 64 empresas, en 1942 se establecieron 57 y en 1944 el número se elevó a 101" (Vásquez, 2001, p. 188). La demanda de trabajo, el mejoramiento de salarios relativos que generó la industrialización y la violencia que caracterizaba el periodo, incrementó el proceso migratorio, convirtiendo a Cali en un punto de atracción demográfica. "Entre 1944 y 1955, Cali pasó de tener 157.813 habitantes a 393.365 ” (p. 201).

El crecimiento desmesurado de la población se enfrentó a una débil infraestructura física y de servicios públicos, lo que contribuyó a la creación de barrios de invasión y problemas de planificación urbana. Todos estos aspectos derivaron en conflictos por la tierra y la vivienda, que se sumaron a las luchas obrero-patronales surgidas por la industrialización.

En este contexto de profundas tensiones políticas y desarrollo socioeconómico se consolidó la salud pública en el país, a través de la creación del Ministerio de Higiene, en 1946, y del Ministerio de Salud Pública en 1953. Todo esto fue posible gracias al proceso de modernización que llevaron a cabo los gobiernos conservadores, como resultado de las misiones de fomento económico y administrativo (Misión Currie), enviadas al país "como requisito para la entrega de ayudas económicas por parte del Banco Mundial y del Banco Internacional para la reconstrucción y el desarrollo" (Henderson, 2006, p. 483).

Una de las principales recomendaciones de la Misión Currie en materia de salud fue "garantizar los mecanismos de salud en Colombia para permitir su conversión hacia una sociedad saludable y sana, es decir productiva y no infectante de forma que el comercio fuese seguro" (Quevedo et al., 2004, p. 332). De este modo se articulaba el desarrollo económico y el bienestar social como una estrategia para mejorar la productividad de la fuerza de trabajo y evitar el "fantasma" de la llegada del comunismo a la región. 
Además de los programas sociales implementados por el Ministerio de Higiene entre 1940 y 1950, es importante destacar la separación de la higiene y la asistencia pública, como actividades centrales para el desarrollo del sector salud en el país -Decreto 408 de febrero de 1947- Gracias a esto las acciones del Estado trascendieron hacia el "desarrollo de servicios de salubridad de cobertura nacional, yendo más allá de la campaña de erradicación, para presionar hacia formas de integración de los servicios preventivos y curativos de los Centros Mixtos de Salud" (Hernández, 2000, pp. 19-20); dichas acciones ya no se limitaban a prestar ayuda a las personas que carecieran de medios de subsistencia o atender a las instituciones de utilidad común.

Segundo, se destaca la organización del Plan Hospitalario por parte del Ministerio de Higiene, expedido a través del Decreto 2554 de la Presidencia de la República, el 28 de julio de 1950, cuyo objeto era atender las necesidades del país a través de la contribución económica y técnica en la construcción de hospitales de diversa índole.

El tercer aspecto tiene que ver con las visitas de dos misiones médicas norteamericanas que recorrieron el país, para evaluar las facultades de Medicina y los hospitales: la Misión Humphrey y la Misión Lapham. La primera, realizada entre el 12 de octubre y el 13 de noviembre de 1948, estuvo encabezada por nueve médicos estadounidenses; una de sus principales recomendaciones fue fundar una nueva facultad de Medicina en la ciudad de Cali. La segunda, realizada entre julio y agosto de 1953, tuvo como objetivo evaluar siete escuelas de Medicina. Ambas plantearon importantes recomendaciones sobre la enseñanza de la Medicina, enfatizando en la práctica clínica, la contratación de docentes de tiempo completo y la creación de especializaciones.

Fueron varios los aspectos que generaron un contexto adecuado para la modernización de las instituciones de salud en el país: la ayuda económica recibida de agencias internacionales, del gobierno norteamericano y de instituciones privadas como la Fundación Rockefeller; el apoyo que tuvieron el Ministerio de Higiene y algunas facultades de Salud en asuntos técnicos y administrativos; y el papel del Estado 
al asumir el fomento, construcción y vigilancia de instituciones hospitalarias. Esto supuso, para el caso particular de la Psiquiatría, la superación de los asilos como instituciones de caridad y asistencia pública y el advenimiento de una nueva infraestructura hospitalaria, soportada en los fundamentos de la ciencia médica para abordar la problemática de la enfermedad mental en el país.

\section{Del Asilo al Hospital}

A diferencia de otras ciudades del país, en donde había instituciones asilares para el tratamiento de los enfermos mentales, en Cali estos eran recluidos en la Casona San Isidro, la cual fungió desde 1937 como correccional de menores, hasta que en 1940 la Asamblea Departamental del Valle, mediante Ordenanza No. 26 de junio de ese mismo año, destinó un presupuesto de $\$ 36.000$.oo para la construcción de un manicomio que permitiera la atención y mejoramiento de los dementes del Departamento (Asamblea Departamental, 1940).

Desde esa misma fecha, el Asilo Meléndez estuvo a cargo de las Hermanas de la Caridad de San Vicente de Paul, las cuales se ocupaban de la asistencia y sostenimiento de los enfermos y, en general, del funcionamiento del Asilo. Como era usual en este tipo de instituciones, la demanda de pacientes superaba la oferta. En 1940, el Asilo San Isidro contaba con la capacidad de albergar 90 pacientes, pero las solicitudes de hospitalización superaban las 500 personas. En lo que respecta a la situación económica, en 1955, el señor Fortunato Aljure, director del Departamento de Higiene, le describía al Dr. Alex Cobo, director del Asilo, su punto de vista sobre la institución (Carta del Doctor Fortunato, 1955):

Usted que ha tenido la generosa preocupación de informarse e interesarse con inspecciones personales del estado actual del Asilo San Isidro, sabe a cabalidad la situación precaria de la institución y la penosa condición en que las limitadas condiciones físicas y económicas, obligan a mantener a sus 200 enfermos. El presupuesto con que cuenta la institución es de \$ 5.800 mensuales, incluyendo 
la nómina de las hermanas, choferes, enfermeros, etc., como solo parte de esta suma se invierte en alimentación, resulta que el presupuesto para la comida es muy inadecuado. Es evidente que con esta suma es imposible alimentar a los enfermos y el enfermo mental goza de buen apetito. Afortunadamente, contribuciones de alimentos por parte de particulares ayudan a calmar el hambre de los alienados. El presupuesto actual no contempla ni permite gastos de droga, haciendo necesario el uso de métodos crueles y anticuados para poder manejar enfermos agitados. Esta falta de elementos técnicos hace posible salvar de la rutina física y psíquica a hombres y mujeres que podrian ser mejorados.

El testimonio del director de Higiene evidencia el abandono en que se encontraban los enfermos mentales en el Asilo, no solo por la falta de recursos para ofrecerles un tratamiento adecuado, sino por la inadecuada planta física con que contaba. Esta situación obligaba permanentemente a trasladar a los enfermos a Pasto, Bogotá o Medellin, lo que suponía altos costos de transporte que eran cubiertos por el erario.

Las críticas que empiezan a hacérsele al Asilo provenian de miembros de diferentes instituciones a nivel nacional y municipal. Dichas críticas, junto con la creación de la Facultad de Salud de la Universidad del Valle, en 1950, generaron lo que March y Olsen (1989, citado por Powell y Dimagio, 1999) denominan oportunidad de cambio: "en ciertos momentos, los recursos acumulados por los autores, sus preferencias o necesidades de modificar sus costos de transacción los conducen a apostar por una transformación mayor en las reglas de juego, en las estrategias de todos o buena parte de los actores relevantes" (p. 27).

Esto permitió, entre otras cosas, la creación de una junta directiva del Asilo, establecida por la Resolución No. 32 de 1951, expedida por el Ministerio de Justicia, con el propósito de llevar a cabo la construcción del hospital psiquiátrico.

La Junta pro construcción convocó a un conjunto de organizaciones y de ciudadanos que se aliaron estructurando 
un campo organizacional, encaminado a generar una nueva infraestructura para la atención de los enfermos mentales de la ciudad, pero también para mejorar el tratamiento y la asistencia clínica a dicha población. En este último aspecto fue clave la creación del Departamento de Psiquiatría en 1956. Las organizaciones que estructuraron dicho campo fueron: la Universidad del Valle, el Ministerio de Salud Pública, la Beneficencia del Valle del Cauca y el Concejo Municipal.

\begin{tabular}{|l|l|}
\hline \multicolumn{1}{|c|}{ INSTITUCIÓN } & \multicolumn{1}{c|}{ REPRESENTANTE } \\
\hline Universidad del Valle & Alex Cobo \\
\hline Ministerio de Salud Pública & Alfonso Bonilla Aragón \\
\hline Beneficencia del Valle & $\begin{array}{l}\text { Germán Holguín Zamorano, Gilberto Gómez } \\
\text { Trujillo }\end{array}$ \\
\hline Consejo Municipal de Cali & Mercedes de Lloreda Garcés \\
\hline
\end{tabular}

Tabla 1. Junta Directiva 1961

Fuente: Orejuela (2014, p. 66)

El Departamento de Psiquiatría se conformó con especialistas formados en el extranjero, principalmente en Estados Unidos. Carlos León, médico psiquiatra ecuatoriano que se incorporó con el Plan Tulane-Colombia, fue el primer jefe del Departamento. Gracias al apoyo de la Universidad de Tulane y de la Fundación Rockefeller que, como se planteó antes, tuvieron un importante papel en la modernización de la salud en el país, muchos médicos viajarían a especializarse a universidades norteamericanas ${ }^{4}$. Esto influenció profundamente el tipo de enfoque implementado tanto a nivel clínico como en la formación de médicos y futuros especialistas. Como lo plantea Adelaida Arboleda (2013, pp. 74-75), para la década de los sesenta:

El Departamento de psiquiatria, por su parte, había incorporado profesores con formación en Harvard y Tulane. Con una actividad gremial importante, con participación en diferentes asociaciones científicas nacionales e internacionales: la Sociedad Colombiana

4 La Psiquiatría norteamericana de mediados del siglo XX, estuvo muy influenciada por el Psicoanálisis. El descubrimiento de fármacos y antidepresivos para el tratamiento de las psicosis hizo que en la década de los sesenta surgiera una Psiquiatría ecléctica que combinaba la Psiquiatría dinámica, el uso de drogas psicoactivas y diferentes tipos de terapias orientadas a la reinserción de los pacientes en la vida cotidiana. 
de Psiquiatría, la World Psychiatric Association, la Academia de Psicoanálisis, la Asociación Americana de Psiquiatría, la Societé Internationale de Psychopatologie de l'expression, la International Epidemiological Association y la America Association for the Advancement of Science, que da cuenta de un capital social importante que posibilitaba el acceso a los grants, pero que además demarcaba líneas teóricas que influenciaban las prácticas locales.

Con las contribuciones realizadas por el gobierno nacional y departamental y las donaciones particulares, se construyó en 1956 la primera etapa del hospital. "Un edificio destinado a la administración, consulta externa, laboratorio, rayos $\mathrm{X}$, botica, servicio social, electroencefalografia, salas de cirugía, enfermería y salas de hospitalización para enfermos quirúrgicos" (Orejuela, 2014, p. 70). En este mismo año, la Junta, con el apoyo del Departamento de Psiquiatría, logró organizar un servicio de consulta externa con ocho consultorios, una oficina de historias clinicas, un archivo y un departamento de servicio social.

La Universidad del Valle fue designada como entidad consejera en aspectos técnicos, decisión que fue fundamental, ya que a través del Departamento no solo acompañó en el diseño y construcción del nuevo hospital, sino que institucionalizó un conjunto de prácticas como el seguimiento riguroso de los enfermos mentales, el uso de los manuales de la APA (DSM-I y DSM-II), para la clasificación y el diagnóstico de enfermedades, así como la realización de tratamientos donde se combinaba el uso de fármacos y el uso de otras estrategias terapéuticas como los electrochoques y la psicoterapia.

Aunque el Hospital tuvo dificultades económicas para continuar con la construcción de la sede, esta se inauguró en 1961. En ese mismo año se lanzó una nueva campaña para recaudar fondos y terminar la edificación de pabellones para pacientes crónicos. Al igual que en otros momentos, se contó con el apoyo de la empresa privada de la región. Los aportes permitieron dotar ocho pabellones para enfermos, cuartos para médicos y empleados y camas para pacientes. 


\section{Las historias clinicas}

Una de las primeras prácticas que se implementaron como resultado del papel que empiezan a tener médicos y estudiantes de la Universidad del Valle en el diagnóstico y el tratamiento de la enfermedad mental en el Asilo San Isidro, fue la elaboración de historias clínicas para cada uno de los enfermos internados o que llegaban por consulta externa. Dichas historias constituyen, en consecuencia, una fuente de primer orden para el estudio del cambio institucional, pues permiten conocer la manera en que se diagnosticaban y clasificaban las enfermedades mentales, así como la estrategia terapéutica que se seguía para enfrentar la enfermedad.

La información disponible en las historias clínicas ofrece datos de la manera cómo funcionaban las instituciones asilares y hospitalarias "y sugieren las características reales de una praxis clínica que no siempre coincidió con los conocimientos o paradigmas imperantes" (Huertas, 2001, p. 8).

A continuación, se abordará el análisis de una muestra de 308 historias clínicas diligenciadas entre los años 1956 y 1970, cuya selección no responde a criterios de representatividad estadística, sino que busca describir la manera en que se institucionalizaron un conjunto de procedimientos médicos, mediante un modo particular de diagnosticar a los enfermos mentales e identificar el tratamiento que recibian. "La institucionalización conlleva procesos por los que los procesos sociales, las obligaciones o las realidades llegan a tomar un estatus de reglas en el pensamiento y la acción sociales" (Meyer y Rowan, 1999, p. 80). En ese orden de ideas, el uso de datos numéricos a través del establecimiento de frecuencias en el estudio, más que mostrar una tendencia fundamentada estadísticamente, busca caracterizar un fenómeno a partir de sus regularidades, pues estas evidencian un determinado uso, con base en la existencia de rutinas y patrones que se convierten en reglas que definen la práctica médica.

Las primeras historias clínicas que reposan en el archivo del hospital psiquiátrico datan del año 1956, aunque se pueden encontrar 
algunas de años anteriores. No es posible determinar, sin embargo, el número total de historias clínicas producidas durante el periodo estudiado, pues de acuerdo con información recibida por el responsable del manejo del archivo, dicho dato, que reposaba en fichas, desapareció del lugar. En términos generales, las historias estaban estructuradas en cuatro partes: la primera, indagaba por datos generales del paciente, como nombre, sexo, edad, domicilio, entidad o persona de quien dependía, etc.; la segunda, abordaba los antecedentes familiares del consultante; la tercera, conformaba la entrevista realizada por el psiquiatra; y la cuarta, incluía un diagnóstico tentativo y el tratamiento a seguir con el paciente. El formato se fue complejizando con el tiempo e incluyó nuevos datos, pero se mantuvieron las preguntas y su estructura en general.

\section{Las historias clinicas del Hospital Psiquiátrico San Isidro 1956-1970}

A continuación, se presentan los principales resultados de la revisión de las 308 historias clínicas consultadas, discriminadas por sexo, edad, estado civil, procedencia, clase de admisión, tipo de atención, diagnóstico y tratamiento. Es relevante mencionar que muchas de las historias contienen fallas de registro, por lo que una importante proporción de datos son referenciados como vacíos o sin información.

\section{Caracterización general de los pacientes: sexo, edad y estado civil}

En la muestra recogida, se registran 169 mujeres y 134 hombres y 5 casos sin información. En lo que respecta a la edad, las historias clínicas se concentran en pacientes que oscilan entre los 10 y los 60 años y más, siendo el principal rango de edad el de 20 a 29 años, con 97 casos; le siguen, en orden, el rango de 10 a 19 con 61 casos, y un tercer rango en importancia, el de 30 a 39 años, con 51 casos. Finalmente, en lo que tiene que ver con el estado civil, se evidencian 129 casos de solteros, 90 de casados, 21 casos en unión libre, 14 viudos y 3 separados; 21 son menores y hay 30 registros sin dato. 


\section{Procedencia de los pacientes}

De las 308 historias registradas, 72 no tienen información sobre la procedencia de los pacientes. En las historias restantes, en un primer lugar se encuentran 97 pacientes procedentes de municipios externos al Departamento del Valle, seguidos de 80 casos de pacientes originarios del Valle, y finalmente, de 58 pacientes que residian en la ciudad de Cali. Esta información resulta interesante, pues muestra la importante cobertura que tenía el hospital; téngase en cuenta que, tal y como se señaló antes, en épocas anteriores los enfermos mentales eran trasladados a las ciudades de Pasto, Medellín y Bogotá.

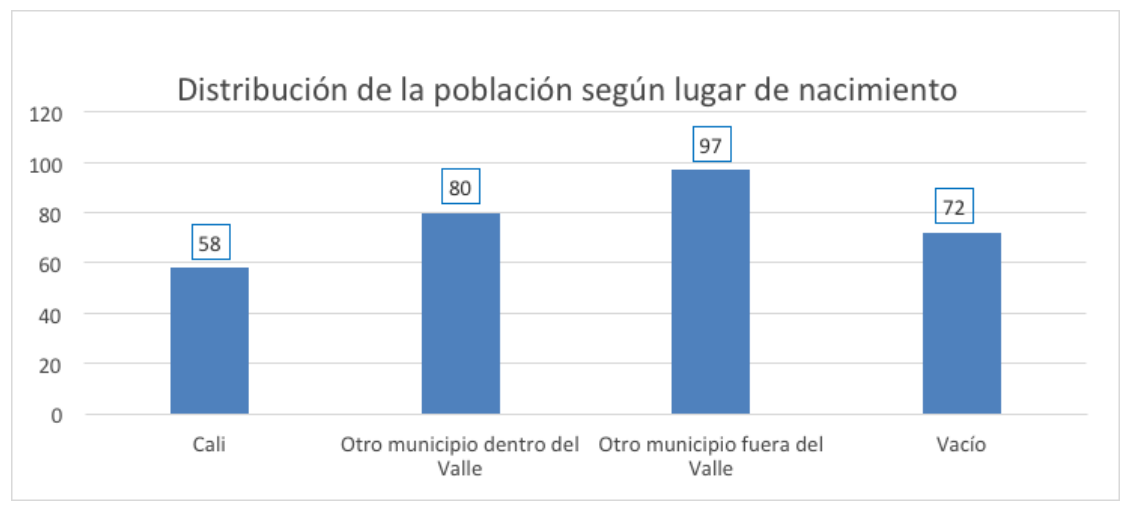

Figura 1

Fuente: Elaboración propia

\section{Clase de admisión}

La revisión realizada permitió establecer que el principal mecanismo de ingreso al hospital es ser llevado a consulta por familiares y amigos: 180 casos; el segundo, ser remitido por policías y extraños, quienes encuentran al paciente en situación de calle y deciden entregarlo a la institución: 24 casos. El tercero, aquel en que los enfermos se presentan voluntariamente a consulta: 15 casos. Finalmente, quienes proceden de otros hospitales, clínicas o médicos: 12 casos. Si bien 
hay 70 historias sin esta información, la ya obtenida contrasta con la que proviene del antiguo Asilo, en donde la mayoría de las personas eran remitidas por las autoridades municipales. No obstante, parece mantenerse como un rezago de dicha institución su concepción de espacio de exclusión y confinamiento, pues, al final, lo que cambian son las personas que llevan a los pacientes para ser recluidos, en este caso familiares y amigos.

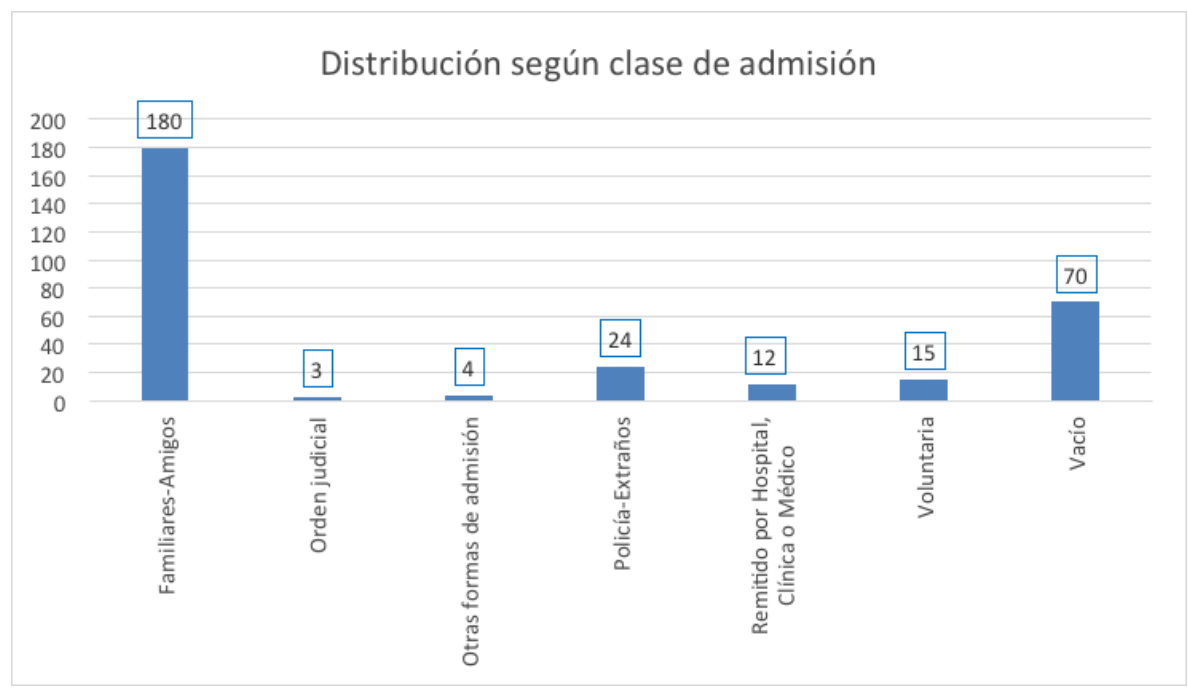

Figura 2

Fuente: Elaboración propia

\section{Tipo de atención}

En lo que respecta a la atención y aunque 158 constituye un importante número de casos sobre los cuales no hay información, los registros obtenidos muestran un cambio fundamental en lo que se refiere a la atención de los pacientes: mientras que en el modelo asilar los enfermos eran hospitalizados por largos periodos, la implementación de la consulta externa desde 1956 posibilita reducir el número 
de personas internadas y ofrece la posibilidad de que los enfermos puedan recuperarse en sus propias casas. Sin embargo, dicha información debe ser contrastada con el número de readmisiones, para de esta forma determinar cuántos de estos enfermos logran realmente ser desinstitucionalizados, es decir, cuántos no requieren volver al hospital, salvo para citas de control.

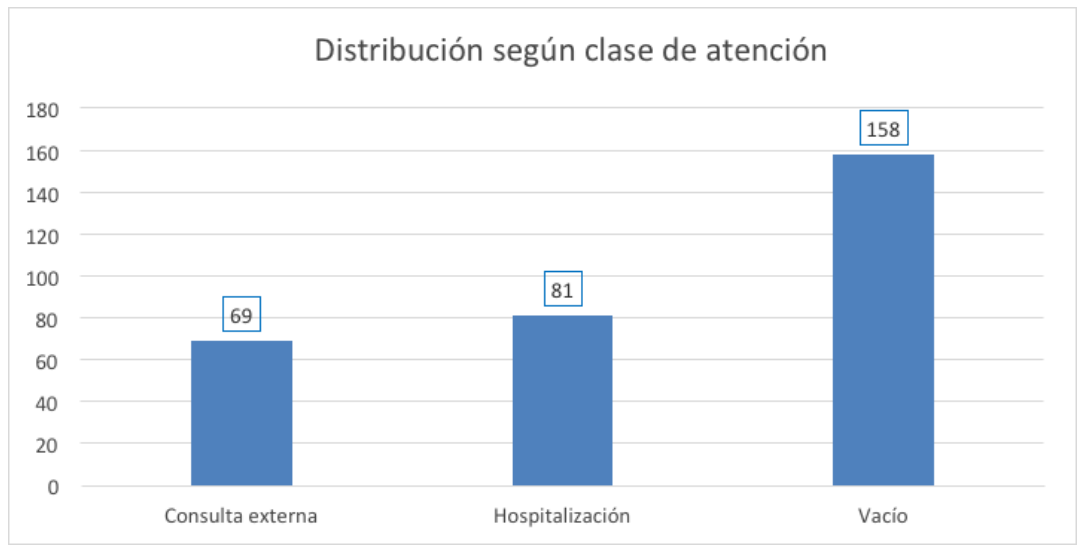

Figura 3

Fuente: Elaboración propia

Al respecto, es clara la Figura 4, pues muestra cómo de las 69 consultas externas realizadas, 58 no llevaron a cabo una nueva consulta; solo 11 personas volvieron: 8 para una consulta, y 3 tuvieron para dos. Lo que parece enfatizar la eficacia de dicho servicio. En lo que se refiere a la hospitalización, la situación difiere un poco: de los 81 casos admitidos, 56 no serían admitidos de nuevo, mientras que 14,8 , 2 y 1 dan cuenta de 1, 2, 3 y hasta 5 readmisiones, respectivamente. Estos datos muestran cómo de las 81 personas internadas, 25 deben ser hospitalizadas de nuevo, lo que equivale a cerca del $31 \%$ del total de hospitalizados durante el periodo estudiado. Estos datos muestran cómo a pesar de nuevos servicios, el hospital no logra desinstitucionalizarse del todo; al contrario, mantiene su carácter manicomial, que 
se expresa en nuevos internamientos para el tratamiento de enfermedades crónicas.

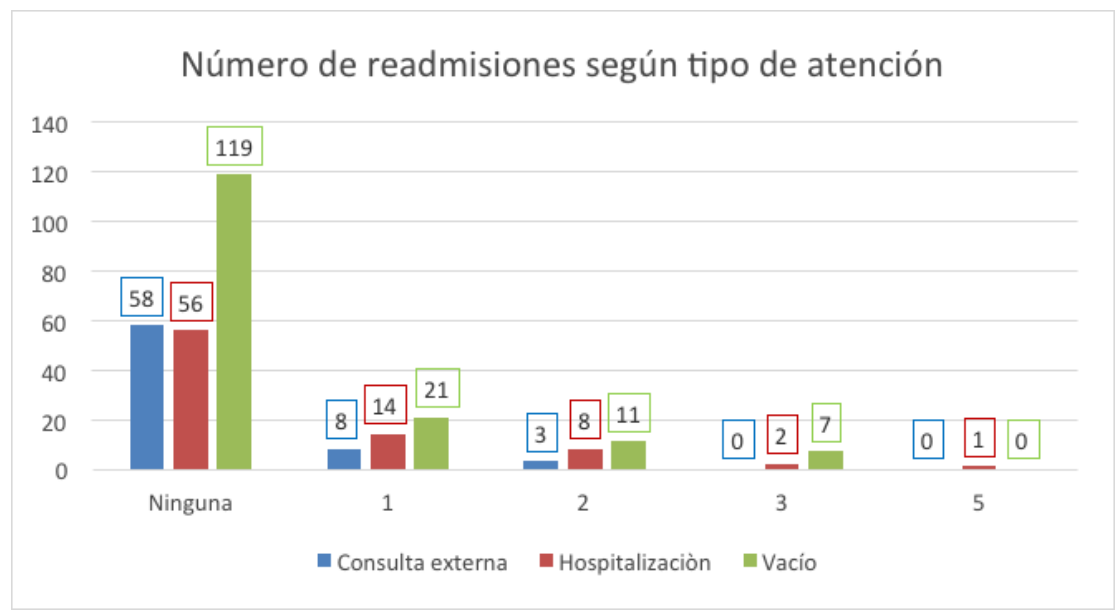

Figura 4

Fuente: Elaboración propia

\section{Diagnósticos}

Las enfermedades más recurrentes durante el periodo estudiado son las reacciones esquizofrénicas, que suman en total 118 casos. En orden le siguen: el síndrome cerebral crónico con 29 casos; otros diagnósticos, entre los que se encuentran las reacciones maniaco depresivas, con 23 casos; la epilepsia con 22 casos; la reacción depresiva con 21 casos; 21 casos son diferidos; 21 sin síntomas y 20 son diagnosticados con reacción angustiosa. Es importante señalar que muchas veces un mismo paciente puede tener dos o más diagnósticos de los ya enunciados. 


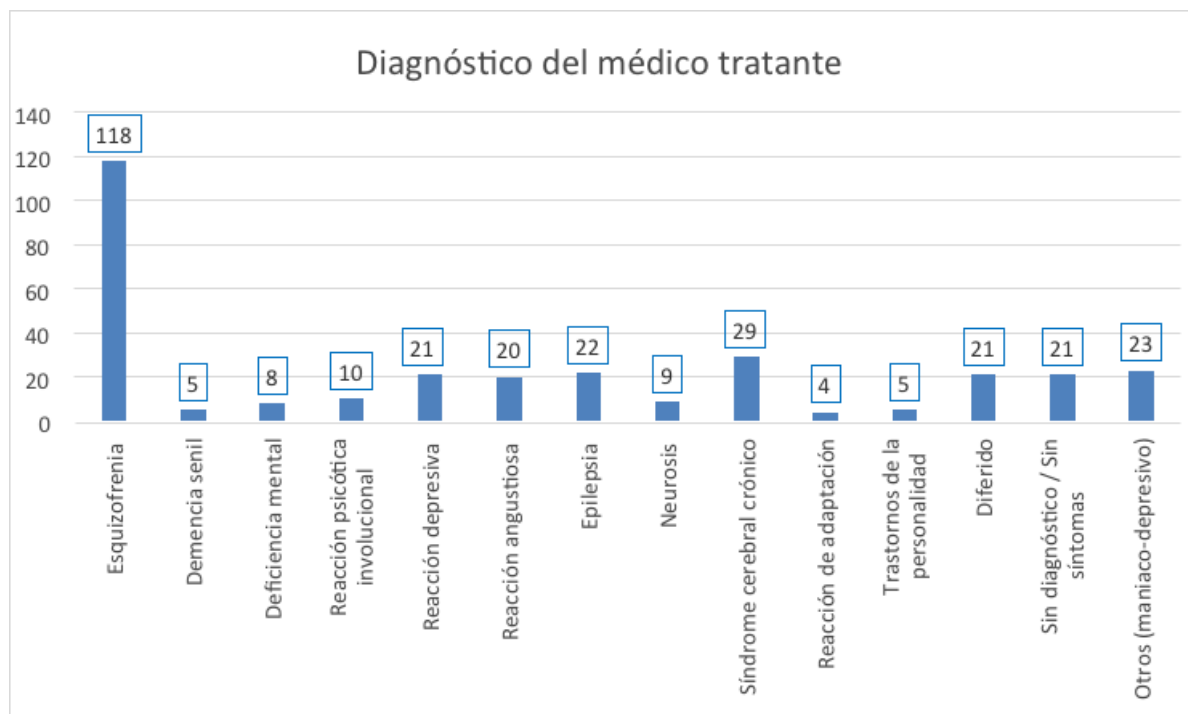

Figura 5

Fuente: Elaboración propia

El término 'reacción' permitió evidenciar la influencia de la visión psicobiológica que caracterizó el DSM I. Según esta visión, los trastornos mentales reflejaban reacciones de la personalidad a factores psicológicos, sociales y biológicos. Los diagnósticos formulados por los psiquiatras muestran el peso que empieza a tener la Psiquiatría psicodinámica y psicoanalítica ${ }^{5}$ de procedencia norteamericana y cómo la vieja utilización de terapias como la insulinoterapia y la malarioterapia, tan en boga en la década de los treinta y cuarenta, serán desplazadas por el uso de fármacos y de terapias de choque.

Para el caso colombiano, Uribe Calad, director del Hospital de Bello (citado por Gutiérrez y Marín, 2012), afirmaba que: "para 1940, los diagnósticos no obedecían a una clasificación médica especializada, porque se ajustaban sólo a la expresión clínica de las manifestaciones morbosas que predominaban en el conjunto de sintomas revelados por el asilado" (p. 210). Entre los principales diagnósticos

Peso que no es similar en otras instituciones psiquiátricas en el país. En el Instituto Psiquiátrico San Camilo, por ejemplo, las enfermedades más recurrentes fueron la epilepsia, que representaba un $22,43 \%$ de las historias consultadas; las esquizofrenias $13,12 \%$; y las oligofrénicas, $12,65 \%$ 
citados en este estudio, se señalaban los siguientes: excitación maniaca por depresión o melancolía, trastornos alimentarios, delirios, megalomanía o delirio de grandeza, matrimonio y desamor, duelo, trabajo, golpes, caquexia demencial y caquexia pelagrosa.

La recurrencia de ciertos diagnósticos en las historias clínicas da cuenta del modo en que se inicia la institucionalización de la práctica médica, pues no solo se inscribe en un enfoque desde el cual se clasifican las enfermedades mentales, sino que, como se señala posteriormente, esto tiene que ver con los tratamientos que se prescriben para enfrentar dichas enfermedades. Esto es evidente cuando se revisa la forma en que se distribuyen los diagnósticos por años, pues se encuentra que entre 1956 y 1964 hay un importante predominio del diagnóstico de esquizofrenia. Después de 1965, es posible constatar una clara disminución de dichos diagnósticos.

Según lo plantea el psiquiatra Roberto Serpa Flores (Escobar, 2009), es posible que una de las razones para la recurrencia del diagnóstico de la esquizofrenia en el periodo estudiado se deba a que en la década de 1950 y principios de 1960 "se usó mucho ese concepto de esquizofrenia y se le trató de aplicar a todos los enfermos psicóticos, es decir, a aquellos que tenían trastornos del pensamiento, afecto y conducta de cierta severidad" (p. 69).

Aunque como lo muestran los registros de las historias clínicas, dicho diagnóstico empieza a cambiar a mediados de la década de los sesenta, la esquizofrenia sigue siendo una de las principales enfermedades identificadas y tratadas en el hospital.

\section{Medicación}

El principal tratamiento recetado por los médicos para enfrentar la enfermedad mental fueron las denominadas drogas psiquiátricas. Aunque durante el periodo estudiado hubo 65 casos en los que no se recetaron ningún tipo de fármacos, los más recurrentes 
fueron antipsicóticos, neurolépticos ${ }^{6}$ y antiepilépticos, como: Largactil (lorpromazina) en 86 casos; Sinogan (Levomepromazina) en 51 casos; Fenergan (Prometazina) en 49 casos; Stelazine (Trifluoperazine) en 36 casos; Gardenal (Fenobarbital) en 31 casos; Stemetil (Prochlorperazine) en 25 casos; Siquil (Trifluopromazine) en 18 casos; y Epamin (Fenitoína) en 18 casos.

La mayoría de estos fármacos son derivados de las fenotiazinas y fueron introducidos en el campo psiquiátrico en la década de los cincuenta, debido a sus virtudes antipsicóticas y neurolépticas, que se descubrieron conforme se utilizaban. Quizá el más importante de estos nuevos medicamentos fue la clorpromazina, cuyo nombre comercial era Largactil, en tanto produjo lo que muchos autores llaman una auténtica revolución farmacológica, pues no solo "introdujo un abordaje selectivo y eficaz para los pacientes esquizofrénicos, sino que abrió las puertas a la síntesis de numerosos fármacos destinados al tratamiento de los trastornos mentales, y, por ende, al inicio de la era psicofarmacológica" (López, Alamo y Cuenca, 2002, p. 80)

Desde el siglo XIX y hasta mediados del siglo XX, los tratamientos de las psicosis eran abordados, sin mayores resultados, por terapias biológicas, tales como: "técnicas de piretoterapia, mediante paludización, aplicación de tuberculina o inducción de abscesos con trementina o las de choque entre las que se destacaban las duchas heladas, los comas insulínicos o cardiazólicos y, sobre todo, la terapia electro convulsiva" (p. 78). Estas terapias se complementaban con tratamiento farmacológico, a través de drogas como el opio, la morfina, la cocaína, el hachís, el hidrato de cloral, entre otras.

Para el caso colombiano, hay una caracterización sobre los métodos de tratamiento, realizada por el doctor Alberto Morales Tobón en su libro El manejo de los enfermos mentales en el manicomio de Medellin (citado por Roselli, 1968, pp. 503-505) en el que identifica cuatro periodos:

6 Utilizados para disminuir la actividad neurológica y lograr el enlentecimiento motor. 
En el primer periodo (1920 a 1937), los métodos de tratamiento eran: 1. Aislamiento y contención (jaulas, grillos, esposas, sacos de fuerza), 2. Abceso de fijación, 3. Arsénico, bismuto y mercurio, 4. Malarioterapia, 5. Piretoterapia, 6. Hipnóticos y sedantes, 7. Dentistería. En el segundo periodo (1938-1949), a las terapias utilizadas en el periodo anterior se le suman la terapia convulsivante por Cardiazol y los electrochoques. El tercer periodo (1950-1955), se caracteriza por nuevos métodos y formas de diagnóstico: insulinoterapia, narcosis prolongada, meloterapia, neuroterapia, psicoterapia, la utilización de la electroencefalografia, entre otros.

De ahí el aporte de los nuevos descubrimientos farmacológicos, pues la clorpromazina y los demás antipsicóticos se convirtieron en la principal alternativa para abordar las esquizofrenias, erradicando paulatinamente los viejos fármacos y las terapias biológicas y de choque; excepto las terapias por electrochoques, que fueron frecuentemente utilizadas como complemento para el tratamiento de las esquizofrenias. Pero quizás uno de los principales aportes de la utilización de drogas psiquiátricas fue "un acortamiento considerable del tiempo de estancia de los nuevos ingresos, así como la salida de algunos crónicos" (Castel, Castel y Lovelle, 1980, p. 91).

\section{Otros tratamientos}

Las drogas psiquiátricas fueron acompañadas de otro tipo de terapias. Entre estas, la de uso más recurrente fue la de electrochoques que, según la revisión, se empleó en 87 casos. Le siguen la terapia ocupacional, con 16 casos, y la psicoterapia, con 10 casos. La Figura 6 evidencia también la disminución en el uso de otras formas terapéuticas, como la insulinoterapia, un caso, y la lobotomía, 2 casos. 


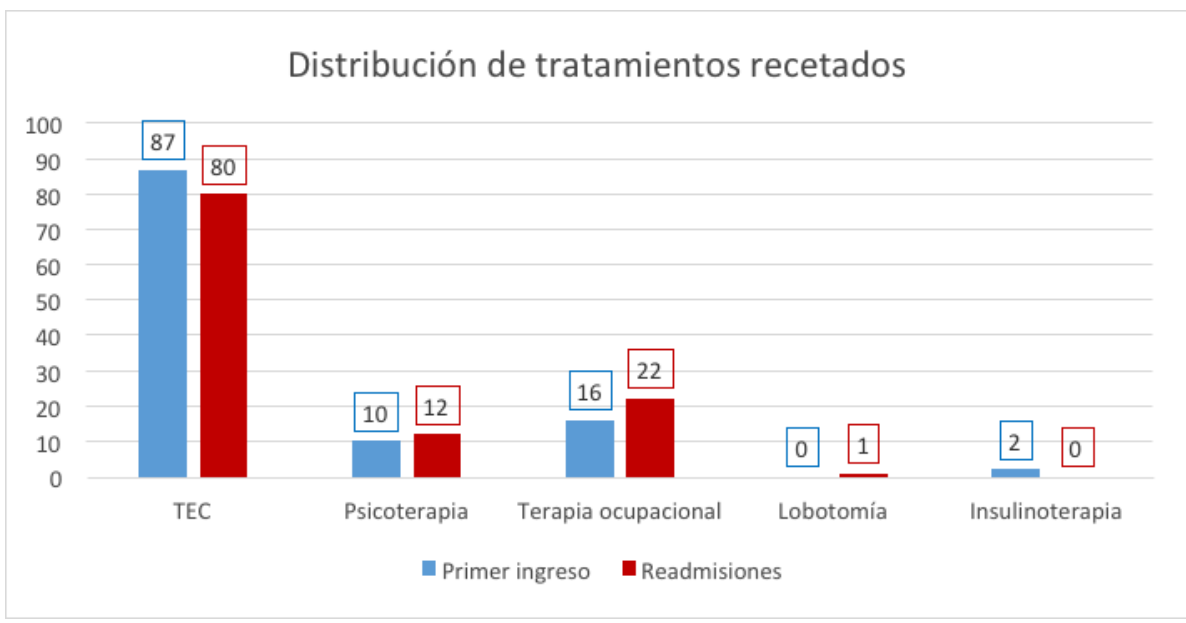

Figura 6

Fuente: Elaboración propia

La Figura 6 también muestra lo correspondiente a los reingresos, ya sea por control en consulta externa o porque son reingresados al hospital, así como la permanencia y reforzamiento en los tratamientos, los cuales dan cuenta de un modo particular de proceder que parece tender a la estandarización de los recursos terapéuticos.

\section{Conclusiones}

El paso del Asilo San Isidro a hospital psiquiátrico moderno supuso la puesta en marcha de un campo organizacional compuesto principalmente por organizaciones públicas: el Concejo municipal, el Ministerio de Salud Pública, la Beneficencia del Valle del Cauca y la Universidad del Valle, las cuales apoyaron a la junta directiva de la institución en el proceso de construcción del nuevo Hospital y posteriormente en su direccionamiento científico.

La conformación de este campo organizacional se llevó a cabo en un momento en que en el país se generaban importantes transformaciones 
en el ámbito de la salud, como parte de una estrategia de modernización administrativa e institucional que se tradujo en visitas de misiones médicas como la Humphrey (1948) y la Lapham (1953), cuyo objetivo fue mejorar la enseñanza de la Medicina en el país, y crear nuevas facultades y especialidades médicas. Todo esto, en un contexto de cambios en la administración del Estado, que dio origen a la creación del Ministerio de Salud Pública, decisión que redundó en el incremento de recursos para el desarrollo hospitalario y en un mayor control público de las instituciones de salud en el país.

A nivel local, se incrementó la presión por parte de diferentes instituciones del orden nacional y departamental, para la construcción de un hospital psiquiátrico que sustituyera el viejo Asilo de dementes, a fin de disminuir los costos que ocasionaba el traslado de pacientes a otras instituciones psiquiátricas. Esto, sumado a la creación de la Facultad de Medicina de la Universidad del Valle en 1950 y del Departamento de Psiquiatria en 1956, generaron las condiciones para que se forjaran alianzas entre las organizaciones y un ambiente propicio para el cambio institucional.

Paralelamente, se estaba generando una importante transformación en el sistema de creencias sobre la salud mental, que influyó profundamente -como lo muestra la revisión de historias clínicas realizadas en este trabajo-, en la práctica médica psiquiátrica. Tres grandes acontecimientos operaron dichos cambios: 1) la revolución farmacológica, que modificó el modo de tratar la enfermedad mental; 2) la creación en 1952, por parte de la Asociación Americana de Psiquiatria, de un manual de clasificación de las enfermedades psiquiatrias (DSM-I); y 3) el advenimiento de nuevas concepciones sobre la enfermedad mental, difundidas por la Organización Mundial de la Salud. Estos tres elementos ofrecieron un nuevo marco de referencia para el diagnóstico y tratamiento de las enfermedades mentales, que incidió en el desarrollo de la especialidad a nivel mundial.

En el caso particular del Hospital Psiquiátrico San Isidro, lo que mostró la revisión de las historias clínicas es que, una vez que el Departamento de Psiquiatría asumió la asesoría 
científica del hospital - su diseño arquitectónico, pasando por la organización administrativa, hasta la implementación de procedimientos y formas de diagnóstico-, estas historias se institucionalizaron de acuerdo con los principios y recomendaciones de la OMS para la enfermedad mental, el manual de diagnósticos DSM-I y la asesoría directa de universidades norteamericanas como Tulane, que se encargaron, además, de la formación de los nuevos especialistas ${ }^{7}$ de Psiquiatría de la Universidad del Valle.

Dicha práctica estuvo sustentada por la introducción de nuevos fármacos para tratar la enfermedad mental, la reutilización de la psicoterapia y la actualización de otras terapias como el electrochoque y la ergoterapia. Estos nuevos tratamientos significaron una clara ruptura con el Asilo para locos que se instauró en 1940, en el que prácticamente no existian historias clínicas, y donde los métodos utilizados para tratar a los enfermos se basaban en su confinamiento, alcanzando un estado deplorable de hacinamiento y maltrato, pues muchos enfermos permanecían amarrados y encadenados.

No obstante, los cambios operados en la práctica médica a través de iniciativas como el Hospital día y la consulta externa no garantizaron el total éxito del nuevo modelo: : aunque fueron muchos los esfuerzos por reducir el número de internos en el Hospital que traía consigo dicho modelo, las readmisiones son una evidencia de los rezagos que sobreviven del modelo asilar en la práctica clínica y de la dificultad para enfrentar el manejo de los pacientes crónicos con las nuevas terapias implementadas. Al respecto, un agravante identificado es el desplazamiento de dichos pacientes a sus contextos.

7 Este último aspecto fue muy importante, porque contribuyó con la profesionalización de la Psiquiatría en la región, a la vez que ayudó a afianzar el nuevo sistema de creencias; los nuevos especialistas, a través de su actividad clínica y formativa, reprodujeron y legitimaron los nuevos saberes y concepciones para abordar la enfermedad mental. 


\section{Referencias}

Arboleda, M. (2013). Relaciones de poder entre agentes en la configuración del campo de la salud mental. Estudio de caso: El programa de psiquiatría comunitaria de la Universidad del Valle, en Cali, Colombia (Tesis de posgrado). Universidad Nacional de Lanús, Buenos Aires, Argentina.

Asamblea Departamental del Valle del Cauca. (1940). Ordenanza No. 26.

Basaglia, F. (1978). Razón, locura y sociedad. México: Siglo XXI.

Bohoslavsky, E. (2005). Instituciones y formas de control social en América Latina 1840-1940. Buenos Aires: Prometeo libros.

Carta del Doctor Fortunato Aljure, Director departamental de higiene, al doctor Alex Cobo. (1955, octubre 25). Correspondencias, Archivo Hospital Psiquiátrico Universitario del Valle, Cali.

Castel, F., Castel, R. y Lovelle, A. (1980). La sociedad psiquiátrica avanzada, el modelo norteamericano. (N. Pérez de Lara Feriada, Trad.) Barcelona: Editorial Anagrama (Obra original publicada en 1979)

Escalante, F. (1992). Ciudadanos imaginarios. México: El Colegio de México.

Escobar, E. (2009). La enfermedad mental en el nororiente de Colombia. Evolución terapéutica en la relación médico-paciente en el Instituto Psiquiátrico San Camilo de Bucaramanga 1953-1967 (Tesis de pregrado). Universidad Industrial de Santander, Bucaramanga, Colombia.

Foucault, M. (1961). Folie et déraison. Histoire de la folie à l'âge classique. Paris: Plon.

Giddens, A. (1979). Central Problems in Social Theory: Action, Structure, and Contradiction in Social Analysis. Berkeley: University of California Press.

Goffman, E. (1972). Internados. Ensayos sobre la situación social de los enfermos mentales. Buenos Aires: Amorrortu editores. 
Gutiérrez, J. y Marín, Y. (2012, julio-diciembre). Poder psiquiátrico, formas clínicas y clasificación de la locura como enfermedad social, en el caso del Manicomio Departamental de Antioquia 1920-1959. Revista Katharsis, 14, 197-224.

Henderson, J. (2006). La Modernización en Colombia. Los años de Laureano Gómez 1889-1965. Medellín: Universidad de Antioquia.

Hernández, M. (2000). La fractura originaria en la organización de los servicios de salud en Colombia 1910-1946. Anuario Colombiano de Historia social y de la Cultura, 27(7), 7-26.

Huertas, R. (2001). Las historias clínicas como fuente para la historia de la psiquiatría: posibles acercamientos metodológicos. Frenia, 1(2), 7-33.

Huertas, R. (2012). Historia cultural de la psiquiatría. (Re) pensar la locura. Madrid: Catarata.

López, F., Álamo, C. y Cuenca, E. (2002). Aspectos históricos del descubrimiento y de la introducción clínica de la clorpromazina: medio siglo de psicofarmacología. Frenia 2(1), 77-107.

Meyer, J. y Rowan, B. (1999). Organizaciones institucionalizadas: la estructura formal como mito y ceremonia. En W. Powell y P. DiMaggio (Eds.), El nuevo institucionalismo en el análisis organizacional (pp. 79-103). México: FCE.

Muñoz, R. (2014). Un enfoque integrador del cambio institucional en los hospitales públicos. En Departamento de Producción y Desarrollo (Ed.), Instituciones y Desarrollo (pp. 405-429). México: UAM-X - CSH.

North, D. (1990). Institutions, Institutional Change and Economic Performance. Cambridge: Cambridge University Press.

Orejuela, D. (2014). La locura en Cali: de una mirada asistencial a una mirada clínica, el caso del Asilo San Isidro 1940-1970 (Tesis de pregrado). Universidad del Valle, Cali, Colombia. 
Powell, W. y DiMaggio, P. (Eds.). (1999). El nuevo institucionalismo en el análisis organizacional (R.R. Reyes, Trad.). México: FCE (Obra original publicada en 1991).

Quevedo, E., Borda, C., Eslava, J., García, C., Guzmán, M. P., Mejía, P. y Noguera, C. (2004). Café y gusanos. Mosquitos y petróleo. El tránsito de la higiene hacia la salud pública en Colombia, 1873-1953. Bogotá: Universidad Nacional de Colombia - Instituto de Salud Pública - Centro de Historia de la Medicina Andrés Soriano Lleras.

Rivas, J. (2003). El neoinstitucionalismo y la revalorización de las instituciones. Reflexión Política, 5(9), 36-46.

Roselli, H. (1968). Historia de la Psiquiatría en Colombia. Bogotá: Editorial Horizontes.

Vásquez, E. (2001). Historia de Cali en el siglo XX, sociedad, economía, cultura y espacio. Cali: Artes gráficas del Valle.

\section{Cómo citar este artículo}

Sánchez Salcedo, J. F. (2017). Diagnóstico y medicación: la práctica médica en el Hospital Psiquiátrico del Valle entre 1956-1970. Universitas Humanística, 83, 331-359. http://dx.doi.org/10.11144/Javeriana.uh83.dmpm 